\title{
Addiction to Tobacco: A War That Begins to Win
}

\author{
Gabriel Miranda Nava* \\ Chief of Neurology of the Hospital Center of the Presidential General Staff, Mexico
}

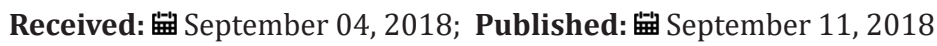

*Corresponding author: Gabriel Miranda Nava, Chief of Neurology of the Hospital Center of the Presidential General Staff, Mexico

\section{Opinion}

For several centuries' humanity has become addicted to tobacco, as a social entrance to an allowed addiction, and that each smoker was charged its respective bill, with ways to die of the most brutal and suffered possible. The complications of nicotine addiction are based on the form of its entry route, which is respiratory due to smoking, and hence the complications of the referred habit, mainly emphysema and lung cancer. The national survey of addictions reveals that in Mexico $18.5 \%$ of the population smokes; that is, almost 20 million people with tobacco habit; almost 50 thousand cases of deaths per year have been reported and that makes us an average of 130 to 140 deaths per day, following the worldwide trend of starring in the number of deaths. How is the death of a chronic smoker patient? It may be due to lung cancer, emphysema or chronic bronchitis, as well as severe sleep apnea, all of which are sponsored by chronic asphyxia, with loss of lung exchange capacity that neither the highest contribution of home or hospital oxygen can compensate; in a few words in the final stage, they agonize, with nothing to do except connect them to an artificial respirator and wait for their slow death; In our country, despite the fact that the number of tobacco addicts has stopped, we will continue to pay the costs of chronic treatment to long-term smokers, say at least 20 more years, as long as the measures adopted take effect.

That without counting other problems such as increased cancer in other parts of the body such as the lip, larynx, urinary bladder, breast, liver, colon, rectum, etc. (ufff, tremendous). Do you want to know more that has been done to decrease your addiction? In our country, legends have been placed in the packs and their tax has been increased and therefore its cost; spaces allowed for smoking have been limited (in enclosed spaces) and advertising and cartels have been placed on a mandatory basis in various public buildings, especially hospitals. The problem is that the fact remains that it is not a generalized prohibition on any enclosed space, and children are still potential victims, and that the tax increase is not as desirable as it could be, but something is something. And it is the only addiction against which there is a real battle, and from which results are expected but in the medium or long term, we will not immediately have the desired answers. Hopefully with alcohol you have the same combat strategies, so that they begin to reduce the cases and complications that are a serious blow to public health.

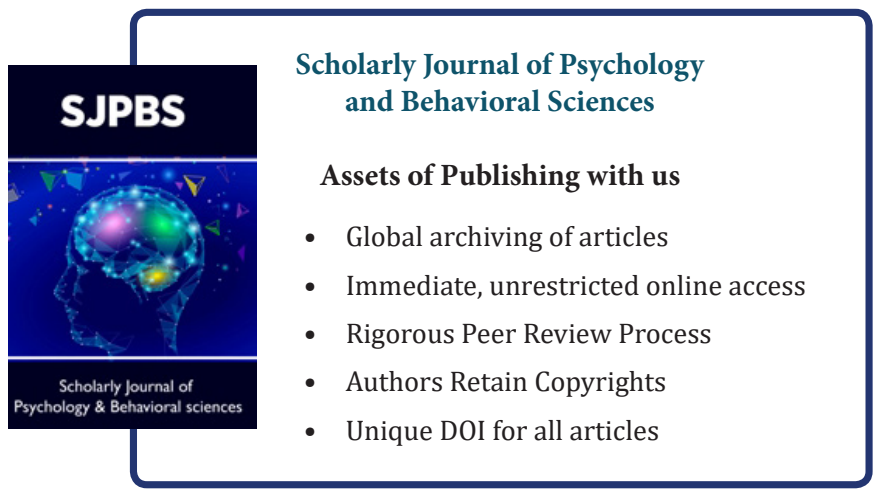

